\title{
The Patient's Gender Influencing the Accuracy of Diagnosis and Proposed Sepsis Treatment in Constructed Cases
}

\author{
Andreas Pikwer, ${ }^{1,2}$ Madeleine Carlsson, ${ }^{3}$ Duraid Abod Mahmoud (D), 1 \\ and Markus Castegren iD $1,3,4$ \\ ${ }^{1}$ Centre for Clinical Research Sörmland, Uppsala University, Uppsala, Sweden \\ ${ }^{2}$ Department of Clinical Sciences at Malmö, Anaesthesiology and Intensive Care, Lund University, Lund, Sweden \\ ${ }^{3}$ Perioperative Medicine and Intensive Care (PMI), Karolinska University Hospital and CLINTEC and FyFa, \\ Karolinska Institutet, Stockholm, Sweden \\ ${ }^{4}$ Department of Medical Sciences, Infectious Diseases, Uppsala University, Uppsala, Sweden
}

Correspondence should be addressed to Markus Castegren; markus.castegren@regionsormland.se

Received 6 March 2020; Revised 16 June 2020; Accepted 23 June 2020; Published 21 July 2020

Academic Editor: Jacek Smereka

Copyright (C) 2020 Andreas Pikwer et al. This is an open access article distributed under the Creative Commons Attribution License, which permits unrestricted use, distribution, and reproduction in any medium, provided the original work is properly cited.

\begin{abstract}
Background. Male sex is an independent risk factor for sepsis development. In addition to immunological gender differences, women less often receive sepsis treatment once diagnosed. Gender differences have also been described in other medical conditions, such as acute coronary syndrome. Aim. To study whether the gender of patients influenced physicians' tendency to suspect sepsis and propose correct initial sepsis treatment in constructed cases. Method. Four cases were constructed to fulfil the sepsis-3 criteria as well as raise clinical suspicions of other common medical differential diagnoses. Two of the cases were drafted in two versions, only differing in the gender of the patient. The two versions were randomly distributed to all clinical physicians in a medical region in Sweden. The responding physicians were asked to state the three most important diagnoses and the three most important initial treatments for each case. If sepsis were among the stated diagnoses together with fluids and antibiotics, the case was considered as correctly identified and initially treated sepsis. Results. 120 hospital physicians answered the cases. In the case the patient was a female, the respondents correctly identified and treated sepsis significantly more often than if the patient was of the male sex (Case 1: $12 / 58$ vs 2/62, $p<0.01$ and Case 2: $25 / 62$ vs $13 / 58, p<0.05$ ). Conclusion. A low proportion of Swedish physicians identified and proposed treatment for sepsis in four constructed cases. In the case the patient strongly mimicked other diagnoses common in the male sex, the male cases were less often correctly identified and treated for sepsis.
\end{abstract}

\section{Introduction}

Sepsis is one of the leading causes of death in the intensive care unit (ICU), with mortality in severe sepsis and septic shock approximating 20\% [1-3]. Sepsis is currently defined as life-threatening organ dysfunction caused by a dysregulated host response to infection [4]. In 2016, the definitions of sepsis and septic shock underwent a revision, known as the sepsis-3 consensus definitions [5]. In the surviving sepsis campaign guidelines, strong recommendations are given to start intravenous antimicrobial treatment within the first hour and fluid resuscitation within the first three hours when a patient fulfills the criteria for sepsis, and in the latter case, when signs of sepsis-induced hypoperfusion are present [6].

Several studies have identified male sex as an independent risk factor for the development of severe infection and sepsis [7-9]. This can in part be attributed to the effects of sex hormones, where androgens have been shown to have immunosuppressive effects in contrast to female sex hormones [9-12]. In the large epidemiological study by Martin et al., men had a higher relative risk (1.28) than women to 
have sepsis [1] even though the incidence of sepsis among women increased more rapidly than among men over the twenty-year period the study covered.

In addition to immunological differences between men and women, it has been shown that sex could influence the management of septic patients. Mikkelsen et al. showed that female sex was associated with a lower usage of early goaldirected therapy [13] in the emergency department. An investigation showed that women with severe sepsis or septic shock were less likely than men to receive antibiotics within three hours from diagnosis [14].

There are sex-associated differences in the management and outcome in several medical emergencies, such as acute coronary syndrome, opioid withdrawal, liver failure, and diabetic ketoacidosis [15-19]. These conditions could, because of resemblance in the clinical presentation, be misinterpreted as sepsis or vice versa.

To further discern the reasons for gender disparities, we aimed to investigate whether the sex of the patient affects the clinicians' decision-making when prioritizing between differential diagnoses. To study this, we used a questionnairebased case study design with the possibility to randomize the sex of the patient with all other variables unchanged. The design allowed the constructed cases to fulfil diagnostic criteria for sepsis as well as strongly resemble other medical conditions. The aim of this investigation was to study whether the sex of the patient influenced the clinical practitioners' ability to diagnose sepsis and order subsequent correct initial treatment, such as fluid resuscitation and antibiotics.

\section{Materials and Methods}

263 physicians with varying experience and from different specialties were asked to anonymously answer a questionnaire containing questions on four constructed patient cases, Figures $1-4$. The questionnaires were sent by e-mail to all physicians working at the County Hospital, Mälarsjukhuset, in the north-western area of the County of Sörmland. The county is in the Mälardalen area of central Sweden. The county's largest hospital is providing specialized health care for 130000 inhabitants as well as being a referral hospital for 140000 additional inhabitants. In the e-mail, the physicians were asked to voluntarily answer the questions and to return the questionnaires by regular mail, to maintain anonymity. The physicians were informed that the purpose of the study was to assess the general level of knowledge of initial treatment to patients with emergency symptoms of critical disease. One month after the initial e-mail, all physicians received a reminding e-mail, asking the ones that had yet not participated in the study to answer the questionnaires.

All four patient cases were constructed to fulfil the diagnostic criteria for severe sepsis according to the international sepsis definitions conference of 2001, so called the sepsis-2 criteria, i.e., $>2$ criteria of systemic inflammatory response syndrome (SIRS) as well as at least one organ dysfunction [20]. All cases also fulfilled the diagnostic criteria for sepsis according to the sepsis-3 criteria [5]. All cases were constructed to show signs of probable sepsis-induced hypoperfusion (hypotension, tachycardia, or hyperlactatemia) where, according to the international guidelines for sepsis and septic shock [6], fluid treatment and early empiric antibiotic treatment are indicated. The cases were also constructed to resemble four different differential diagnoses, i.e., acute coronary syndrome, opioid withdrawal, liver failure, and diabetic ketoacidosis. The rationale for this was that a number of missed sepsis diagnoses in several different patients were discussed in morbidity and mortality rounds at the ICU, where patients had been primarily treated for other conditions than sepsis but were later rediagnosed as sepsis due to positive growth in blood cultures. The four aforementioned conditions were identified as differential diagnoses where sepsis easily could be missed. The four cases were nonauthentic, but inspired by patients that had primarily been diagnosed for other conditions than sepsis. The authors of this article are clinical experts and researchers in emergency medicine and intensive care medicine and jointly constructed the four cases. The cases were constructed in two versions, $\mathrm{A}$ and $\mathrm{B}$. The versions differed in only one aspect, i.e., the sex of the patients in patient Cases 1 and 2. In Cases 3 and 4, the sex of the patients was the same in both versions. In version $A$ of the questionnaire, the patient in Case 1 was a male, whereas the patient in Case 2 was a female. In version $\mathrm{B}$, the patients in Cases 1 and 2, were female and male, respectively. In both versions, the patient in Case 3 was female and male in Case 4. Which version of the questionnaire that has to be sent out to each participant was randomly chosen. All cases can be seen in Figures 1-4.

In each case, the participants were asked to identify the three most probable and important-to-treat differential diagnoses and propose the three most important primary measures to be carried out and to assess at which level of care the patient should initially be treated, i.e., either at a general ward, at a high-dependence ward, or at an intensive care unit. In addition to answering the questions above, the participants were asked to give their corresponding age span (20-30, 30-40, 50-60, or 60+ years), their sex, and their position (intern, resident, consultant, or senior consultant), as well as their field of medical specialty.

2.1. Ethics. According to the Swedish legislation, the study did not need ethical clearance by the Swedish ethical review authority since all cases were constructed and nonauthentic and all participants were asked to answer the cases anonymously.

\subsection{Treatment of Data and Statistics. All answers were} transferred to a data sheet after the termination of the study. A variable named "correct identification and initial sepsis treatment" was constructed in the data sheet. If a participant had identified sepsis as one of the three most important and probable diagnoses as well as proposed antibiotics and fluid administration to be among the three most important measures to be carried out in a case, that case was given the value 1 on "correct identification and initial sepsis treatment." Failure to either identify sepsis or propose antibiotics or fluid treatment yielded the value 0 . 


\section{Case 1}

A 60-year-old male (version A)/female (version B) presents at the ER with chest pain and pain in the left leg. The chest pain started three hours ago, whereas the leg has been hurting since the pain woke the patient up, seven hours ago. The patient has tried prescribed nitroglycerin but with no symptom relief. The patient breathes rapidly and reports anxiety. In the prior medical history, the patient reports peripheral arterial insufficiency and CABG surgery for three-vessel coronary disease, five years ago. The patient has, until now, not experienced any angina pectoris since the CABG surgery.

Prescribed medication: nitroglycerin, acetylsalisylic acid, simvastatin, ACE-inhibitor.

Status: Anxious and oriented to time, space, and person. No cardiac murmurs. Bilateral basal lung crepitations. No palpable pulses in the left tibialis posterior or in the left dorsalis pedis arteries. The left leg is pale below the knee. There is an open, moist wound on the left foot with necrotic tissue around the wound. ECG: LBBB, atrial fibrillation.

Vital signs: Temp. $38.5^{\circ} \mathrm{C}$. Respiratory rate 23 . BP $95 / 50$. Heart rate 140 . Arterial oxygen saturation $87 \%$, rises to $96 \%$ after $10 \mathrm{~L} \mathrm{O}_{2}$ on face mask.

Lab: Hemoglobin $13.0 \mathrm{~g} \times \mathrm{dL}^{-1}$. Leukocyte count $17.9 \times 10^{9} \times \mathrm{L}^{-1}$. C-reactive protein $146 \mathrm{mg} \times \mathrm{L}^{-1}$. Sodium $135 \mathrm{mmol} \times \mathrm{L}^{-1}$. Potassium $3.8 \mathrm{mmol} \times$ $\mathrm{L}^{-1}$. Creatinine $1.9 \mathrm{mg} \times \mathrm{dL}^{-1}$.

Figure 1: Case 1 .

\section{Case 2}

On the ward, you see a 35-year-old male (version B)/female (version A) who had emergency surgery two days ago for a ruptured appendix. The patient has a history of substance abuse with intravenous opoids and alcohol. The patient is seropositive for hepatitis $\mathrm{C}$. The patient has during the day become aggressive and disoriented. The patient is not satisfied with the care provided and wants to go home. The patient wants more pain relief and something to "cool down".

Status: Agitated and awake. The skin is moist. The patient does not allow any examination of the heart and lungs. The patient cannot relax the abdominal muscles during abdominal palpation.

Vital signs: Temperature $38.5^{\circ} \mathrm{C}$. Respiratory rate 25. BP 170/80. Heart rate 110 .

Lab: Hemoglobin $16.0 \mathrm{~g} \times \mathrm{dL}^{-1}$. Leukocyte count $25 \times 10^{9} \times \mathrm{L}^{-1}$. C-reactive protein $280 \mathrm{mg} \times \mathrm{L}^{-1}$. Sodium $129 \mathrm{mmol} \times \mathrm{L}^{-1}$. Potassium $3.6 \mathrm{mmol} \times \mathrm{L}^{-1}$. Creatinine $2.5 \mathrm{mg} \times \mathrm{dL}^{-1}$. Glucose $250 \mathrm{mg} \times \mathrm{dL}^{-1}$.

FIGURE 2: Case 2.

\section{Case 3}

At $04.30 \mathrm{am}$, a 46-year-old female (version A and B) presents at the ER, transported with an ambulance. The accompanying husband reports that the patient has breast cancer and is currently undergoing cytostatic treatment. The last treatment was given two weeks ago. There are no other illnesses in the medical history. The husband was wakened when the patient began "shaking" in bed. She was breathing irregularly and snored noisily. The husband phoned the EMS when he was unable to receive contact with the patient.

Status: The patient opens her eyes and can say her name, but is disoriented to time and place. She denies pain or discomfort. She has no signs of cardiac decompensation, but she is icteric.

Vital signs: Temperature $34.2^{\circ} \mathrm{C}$. Respiratory rate 25. BP 80/60, rises to $100 / 50$ after $1 \mathrm{~L}$ of warm Ringer's acetate. Heart rate 120 . Oxygen saturation $92 \%$ on $2 \mathrm{~L} \times \mathrm{min}^{-1} \mathrm{O}_{2}$.

Lab: Hemoglobin $12.0 \mathrm{~g} \times \mathrm{dL}^{-1}$. Leukocyte count $1.5 \times 10^{9} \times \mathrm{L}^{-1}$. C-reactive protein $30 \mathrm{mg} \times \mathrm{L}^{-1}$. Creatinine $3.5 \mathrm{mg} \times \mathrm{dL}^{-1}$. Bilirubin $12 \mathrm{mg} \times \mathrm{dL}^{-1}$. Glucose $28 \mathrm{mg} \times \mathrm{dL}^{-1}$.

Figure 3: Case 3.

The frequencies of correct identification and initial sepsis treatment, sepsis among the three most important diagnoses, and proposed fluid and antibiotics among the three most important measures to be carried out, as well as ICU as the appropriate level of care, were compared between the questionnaire versions $\mathrm{A}$ and $\mathrm{B}$ and analysed for differences with $\mathrm{Chi}^{2}$ tests and Fisher's exact tests, where appropriate. A $p$ value of $<0.05$ was considered statistically significant. Statistica (Statsoft, Tulsa, OK, USA) was used in the statistical calculations. 
Case 4

A 53-year-old male (version A and B) presents at the ER as a referral from his family physician under the suspicion of "Debuting diabetes mellitus with ketonic acidosis?". The referral note further says: "Well known patient with difficult-to-control arterial hypertension. Presented at my office two days ago with a reddening rash on his left forearm which I assessed as erysipelas. I prescribed Penicillin orally $1 \mathrm{~g}, 3$ times a day for ten days. The patient presents today complaining that he feels very tired and has abdominal ache. A urine test stick shows $3+$ for ketones. His blood glucose is $350 \mathrm{mg} \times \mathrm{dL}^{-1}$.

Status: Fully awake and oriented. The abdomen is tender but soft. Normal bowel sounds. Heart and lungs have normal sounds.

Vital signs: Temperature $38.5^{\circ} \mathrm{C}$. Respiratory rate 30 . BP 100/80. Oxygen saturation $96 \%$.

Lab: Hemoglobin $15.5 \mathrm{~g} \times \mathrm{dL}^{-1}$. Leukocyte count $25 \times 10^{9} \times \mathrm{L}^{-1}$. Platelets $100 \times 10^{9} \times \mathrm{L}^{-1}$. C-reactive protein $100 \mathrm{mg} \times \mathrm{L}^{-1}$. Glucose $380 \mathrm{mg} \times \mathrm{dL}^{-1}$. pH 7.22. PO2 $10 \mathrm{kPa}$. PCO2 $2.8 \mathrm{kPa}$. BE $-18 \mathrm{mmol} \times \mathrm{L}^{-1}$. Lactate $3.5 \mathrm{mmol} \times \mathrm{L}^{-1}$.

Figure 4: Case 4.

\section{Results}

In total, 120 participants answered a questionnaire and returned them anonymously by regular mail, rendering a response frequency of $45.7 \%$. The participants were evenly distributed among the age groups, position, and medical specialties, Table 1 . When the participants that had completed versions A and B were compared, no significant differences concerning the age span, position, or medical specialty were seen, Table 1 .

3.1. Case 1. The most common diagnoses proposed by the participants in both $\mathrm{A}$ and $\mathrm{B}$ versions were myocardial ischemia, followed by pulmonary embolism and acute arterial thromboembolism. These three diagnoses were proposed by 93, 77, and $69 \%$ of all participants, respectively, as being among the three most important and probable differential diagnoses. Only 36/120 of the participants had proposed sepsis as one of the three most important diagnoses.

A significantly higher part of the participants that had answered version B of the questionnaire, i.e., where the patient was of female sex, had correctly identified sepsis as an important differential diagnosis as well as proposed both antibiotics and fluids among the three most important initial therapeutic measures, Table 2. In the answers of version B, the participants had proposed that antibiotics and fluids belonged to the three most important initial measures to a higher degree than in version A, Table 2. Approximately, $60 \%$ proposed ICU as the appropriate level of care with no differences between versions $\mathrm{A}$ and $\mathrm{B}$ of the questionnaire.

3.2. Case 2. The most common diagnoses proposed by the participants were sepsis followed by drug withdrawal symptoms and postoperative complications (i.e., postoperative bleeding, postoperative infection, bowel perforation, and ileus). These three diagnoses were proposed by 88, 73, and $42 \%$ of all participants, respectively, as being among the three most important and probable differential diagnoses. There were no differences when the participants' answers regarding diagnosis between versions $\mathrm{A}$ and $\mathrm{B}$ were compared.

Compared to the participants that had answered version $B$, a significantly higher part of the participants that had
TABle 1: The distribution of the participants answering the two versions of the questionnaire with regard to sex, age, seniority, and medical specialty.

\begin{tabular}{lccc}
\hline Version & $\mathrm{A}$ & $\mathrm{B}$ & $\mathrm{p}$ \\
\hline$n(\%)$ & 62 & 58 & \\
Male sex $n(\%)$ & $34(55)$ & $31(51)$ & 0.66 \\
Responder age span $n(\%)$ & & & \\
$\quad 20-30$ & $14(23)$ & $13(22)$ & 0.89 \\
$30-40$ & $21(34)$ & $24(41)$ & 0.43 \\
$40-50$ & $12(19)$ & $12(21)$ & 0.78 \\
$50-60$ & $10(16)$ & $5(9)$ & 0.25 \\
$\quad 60+$ & $1(2)$ & $1(2)$ & 1.00 \\
Responder position $n(\%)$ & & & \\
$\quad$ Intern & $18(29)$ & $13(22)$ & 0.38 \\
$\quad$ Resident & $20(32)$ & $26(45)$ & 0.14 \\
Consultant/senior consultant & $24(39)$ & $17(29)$ & 0.25 \\
Medical specialty $n$ (\%) & & & \\
$\quad$ Internal medicine specialties & $19(31)$ & $18(31)$ & 0.91 \\
Surgical specialties & $10(16)$ & $10(17)$ & 0.88 \\
$\quad$ Infectious diseases & $3(5)$ & $4(7)$ & 0.64 \\
$\quad$ Anaesthesia and intensive care & $11(18)$ & $10(17)$ & 0.89 \\
$\quad$ Psychiatry & $2(3)$ & $2(3)$ & 1.00 \\
\hline
\end{tabular}

Data are presented as $n(\%)$. The $p$ values are results from $\mathrm{Chi}^{2}$ tests and Fisher's exact tests, where appropriate.

answered version $\mathrm{A}$ of the questionnaire, i.e., where the patient was of the female sex, had correctly identified sepsis as an important differential diagnosis as well as proposed both antibiotics and fluids among the three most important initial therapeutic measures, Table 2. In the answers of version $A$, the participants had proposed that fluids belonged to the three most important initial measures to a higher degree than in version B, Table 2. Approximately, $52 \%$ had proposed ICU as the appropriate level of care with no differences between versions $A$ and $B$ of the questionnaire.

3.3. Control Cases, 3 and 4. In Case 3, the most common diagnosis proposed by the participants was sepsis, followed by brain metastases associated with epilepsy and liver failure. These three diagnoses were proposed by 85,40 , and $39 \%$ of all participants, respectively, as being among the three most important and probable differential diagnoses. Approximately, $77 \%$ had proposed ICU as the appropriate level of 
TABle 2: Distribution of the participants' answers to the two versions of the questionnaire.

\begin{tabular}{|c|c|c|c|c|c|c|c|c|c|c|c|c|}
\hline \multirow[b]{2}{*}{ Version } & \multicolumn{3}{|c|}{ Case 1} & \multicolumn{3}{|c|}{ Case 2} & \multicolumn{3}{|c|}{ Case 3} & \multicolumn{3}{|c|}{ Case 4} \\
\hline & $\underset{(\text { male })}{\mathrm{A}}$ & $\begin{array}{c}\mathrm{B} \\
\text { (female) }\end{array}$ & $p$ & $\begin{array}{c}\mathrm{A} \\
\text { (female) }\end{array}$ & $\underset{\text { (male) }}{\mathrm{B}}$ & $p$ & $\underset{\text { (female) }}{\mathrm{A}}$ & $\begin{array}{c}\mathrm{B} \\
\text { (female) }\end{array}$ & $p$ & $\underset{(\mathrm{male})}{\mathrm{A}}$ & $\begin{array}{c}\mathrm{B} \\
\text { (male) }\end{array}$ & $p$ \\
\hline$n$ & 62 & 58 & & 62 & 58 & & 62 & 58 & & 62 & 58 & \\
\hline $\begin{array}{l}\text { Correct identification and initial } \\
\text { sepsis treatment }\end{array}$ & $2(4)$ & $12(21)$ & $<0.01$ & $25(40)$ & $13(22)$ & $<0.05$ & $22(35)$ & $25(43)$ & 0.40 & $20(32)$ & $20(34)$ & 0.79 \\
\hline Sepsis among differential diagnoses & $16(26)$ & $20(34)$ & 0.30 & $52(84)$ & $54(93)$ & 0.10 & $51(82)$ & $51(88)$ & 0.36 & $42(68)$ & $44(76)$ & 0.33 \\
\hline $\begin{array}{l}\text { Proposed fluid among initial } \\
\text { treatments }\end{array}$ & $15(24)$ & $24(41)$ & $<0.05$ & $35(56)$ & $21(36)$ & $<0.05$ & $31(50)$ & $37(64)$ & 0.12 & $45(73)$ & $41(71)$ & 0.81 \\
\hline $\begin{array}{l}\text { Proposed antibiotics among initial } \\
\text { treatments }\end{array}$ & $5(8)$ & $16(28)$ & $<0.05$ & $41(66)$ & $34(59)$ & 0.33 & $35(56)$ & $33(57)$ & 0.91 & $32(52)$ & $29(50)$ & 0.83 \\
\hline $\begin{array}{l}\text { Proposed ICU as the appropriate } \\
\text { level of care }\end{array}$ & $37(60)$ & $34(58)$ & 0.97 & $35(56)$ & $27(47)$ & 0.51 & $50(80)$ & $42(72)$ & 0.25 & $34(55)$ & $37(64)$ & 0.16 \\
\hline
\end{tabular}

Data are presented as $n$ (\%). The $p$ values are results from $\mathrm{Chi}^{2}$ tests and Fisher's exact tests, where appropriate.

care. In total, $39 \%$ of the physicians proposed sepsis as an important differential diagnosis and proposed antibiotics and fluid resuscitation among the most important initial therapeutic measures, with no differences between the participants that answered the version $\mathrm{A}$ or $\mathrm{B}$ of the questionnaire.

In Case 4, the most common diagnoses proposed by the participants were diabetic ketoacidosis, sepsis, and infection. These three diagnoses were proposed by 100,72 , and $25 \%$ of all participants, respectively, as being among the three most important and probable differential diagnoses. Approximately, 59\% had proposed ICU as the appropriate level of care. In total, $55 \%$ of the physicians proposed sepsis as an important differential diagnosis and proposed antibiotics and fluid resuscitation among the most important initial therapeutic measures, with no differences between the participants that answered the version $\mathrm{A}$ or $\mathrm{B}$ of the questionnaire.

\section{Discussion}

The current study found that, keeping all other factors the same, just changing the sex of the patients in constructed cases affected the physicians' ability to correctly identify sepsis and suggest correct initial sepsis treatment. In the case of a female patient, a correct sepsis diagnosis and initial treatment was more likely.

The reason for this difference is not obvious and contradicts previous clinical observations $[1,13,14]$. In this study, all the cases showed symptoms of sepsis, but the scenarios also strongly mimicked other medical emergencies. A possible explanation is that the physicians' estimation of the probability of the differential diagnoses such as acute coronary syndrome and opioid withdrawal depended on the sex of the patient, and swayed the physicians to focus their initial treatment suggestions on those diagnoses.

The symptoms described in Case 1 strongly suggested acute coronary syndrome. It is well established that there are sex disparities in the incidence [21], presentation [22], diagnostics [23], and treatment of acute coronary syndrome. Possibly, this knowledge influenced the physicians' clinical judgement. Thus, increasing the probability that a male, compared to a female patient, with signs of both acute coronary syndrome and sepsis will be treated for acute coronary syndrome rather than sepsis.

The symptoms described in Case 2 indicated opioid withdrawal. Opioid addiction is less common in females compared to men [18], and men have a higher rate of receiving treatment for opioid withdrawal [24]. This may have swayed the physicians to suspect and focus treatment on opioid withdrawal in the case with the male patient compared to the case with the female patient. Thus, making sepsis a less likely diagnosis for men. However, not studied in the present investigation, one could speculate that the result could have been opposite if one of the constructed cases would have resembled medical conditions more common in the female sex, e.g., intoxication by oral sedatives.

Although both Cases 1 and 2 described patients with sepsis, the differential diagnoses of acute coronary syndrome and opioid withdrawal, respectively, were more often proposed than sepsis by the physicians included in the study. A minority of physicians made the correct sepsis diagnosis. The participating physician was most often not specifically trained in emergency medicine, infectious disease medicine, or intensive care medicine. This probably contributed to the poor rate of correct sepsis diagnosis and subsequent initial treatment. This study was not designed to study the effect of knowledge in sepsis treatment and hence, no such analyses were performed. However, the doctors working at the emergency room in the Swedish healthcare system are often inexperienced doctors from different specialties, seldom specialized in emergency care. This increases the applicability of this study in the Swedish setting. If the cases would have been more obvious regarding the symptomatology of sepsis or if the participating physicians would, to a higher degree, have been trained in sepsis management, the results could have been different. It might be the participating physicians' low rate of sepsis management training and the cases' resemblance to other diagnoses that paradoxically resulted in the higher rate of sepsis diagnoses in female patients. This could explain our diverging results compared to the clinical observations that males more often suffer sepsis compared to females [1] and that females are less likely 
to receive correct sepsis treatment $[13,14]$. A small number of specialist physicians in clinical psychiatry were included in the study. The rationale for including psychiatrists was that in Case 2, the presentation resembled opioid withdrawal. One possible proposed initial measure could have been referral to psychiatry. The small amount of psychiatrists included did not influence the result (data not shown).

The study has several limitations. First, a questionnairebased study can obviously never be totally representative of how the physicians would have reasoned in actual patients. Second, the anonymous design of the study made it impossible to approach the physicians who did not respond, thus leading to a fairly high share of nonresponders. Third, the study is a fairly small single-centre study which makes it hard to draw strong conclusions.

The design of the study was an innovative approach to be able to study the effect of gender. The clinical implication of the study is that clinical physicians should be aware that clinical decision-making can be influenced by gender, especially when assessing patients with sepsis and clinical conditions resembling septic symptoms. Gender medicine in conjunction with knowledge of septic symptoms and sepsis management should be an essential part in various stages of training for physicians, as well as in the continuing learning process after achieved medical specialty certification.

It would be of interest to conduct similar studies in other healthcare systems than the system currently studied and also to study whether the effect could be replicated in a population of physicians with a higher level of training in emergency medicine and sepsis management. Further, it would be of interest to study whether the observed effects of gender also exist in other diagnoses than sepsis.

\section{Conclusion}

In this questionnaire-based case study, a low proportion of Swedish physicians identified sepsis as an important differential diagnosis in four constructed cases. Female sex in patients rendered a higher probability for correctly identifying sepsis as an important differential diagnosis and proposing adequate initial treatment for sepsis. The study design is well suited for studying the way differential diagnoses are formed.

\section{Data Availability}

Data are available upon request to the corresponding author.

\section{Conflicts of Interest}

The authors declare that they have no conflicts of interest.

\section{References}

[1] G. S. Martin, D. M. Mannino, S. Eaton, and M. Moss, "The epidemiology of sepsis in the United States from 1979 through 2000," New England Journal of Medicine, vol. 348, no. 16, pp. 1546-1554, 2003.

[2] S. Jacobson, G. Johansson, and O. Winsö, "Primary sepsis in a university hospital in northern Sweden: a retrospective study,"
Acta Anaesthesiologica Scandinavica, vol. 48, no. 8, pp. 960967, 2004.

[3] A. M. Higgins, S. L. Peake, R. Bellomo et al., "Quality of life and 1-year survival in patients with early septic shock," Critical Care Medicine, vol. 47, no. 6, pp. 765-773, 2019.

[4] C. W. Seymour, V. X. Liu, T. J. Iwashyna et al., "Assessment of clinical criteria for sepsis," JAMA, vol. 315, no. 8, pp. 762-774, 2016.

[5] M. Singer, C. S. Deutschman, C. W. Seymour et al., "The third international consensus definitions for sepsis and septic shock (Sepsis-3)," JAMA, vol. 315, no. 8, pp. 801-810, 2016.

[6] A. Rhodes, L. E. Evans, W. Alhazzani et al., "Surviving sepsis campaign: international guidelines for management of sepsis and septic shock: 2016," Intensive Care Medicine, vol. 43, pp. 304-377, 2017.

[7] P. J. Offner, E. E. Moore, and W. L. Biffl, "Male gender is a risk factor for major infections after surgery," Archives of Surgery, vol. 134, no. 9, pp. 935-938, 1999.

[8] B. G. Szabo, R. Kiss, K. S. Lenart et al., "Clinical and microbiological characteristics and outcomes of communityacquired sepsis among adults: a single center, 1-year retrospective observational cohort study from Hungary," $B M C$ Infectious Diseases, vol. 19, p. 584, 2019.

[9] M. K. Angele, S. Pratschke, W. J. Hubbard, and I. H. Chaudry, "Gender differences in sepsis," Virulence, vol. 5, no. 1, pp. 12-19, 2014.

[10] S. Sakiani, N. J. Olsen, and W. J. Kovacs, "Gonadal steroids and humoral immunity," Nature Reviews Endocrinology, vol. 9, no. 1, pp. 56-62, 2013.

[11] R. C. Gaillard and E. Spinedi, "Sex- and stress-steroids interactions and the immune system: evidence for a neuroendocrine-immunological sexual dimorphism," Domestic Animal Endocrinology, vol. 15, no. 5, pp. 345-352, 1998.

[12] G. Zandman-Goddard, E. Peeva, and Y. Shoenfeld, "Gender and autoimmunity," Autoimmunity Reviews, vol. 6, no. 6, pp. 366-372, 2007.

[13] M. E. Mikkelsen, D. F. Gaieski, M. Goyal et al., "Factors associated with nonadherence to early goal-directed therapy in the ED," Chest, vol. 138, no. 3, pp. 551-558, 2010.

[14] T. E. Madsen, J. Simmons, E. K. Choo, D. Portelli, A. J. McGregor, and A. M. Napoli, "The DISPARITY Study: do gender differences exist in Surviving Sepsis Campaign resuscitation bundle completion, completion of individual bundle elements, or sepsis mortality?" Journal of Critical Care, vol. 29, pp. 473.e7-473.e11, 2014.

[15] T. Romero, P. Velez, D. Glaser, and C. X. Romero, "Do gender and race/ethnicity influence acute myocardial infarction quality of care in a hospital with a large Hispanic patient and provider representation?" Cardiology Research and Practice, vol. 2013, Article ID 975393, 7 pages, 2013.

[16] D. U. Himmelstein, "Sex differences in alcohol-related disease at a county hospital," International Journal of the Addictions, vol. 18, no. 1, pp. 125-131, 1983.

[17] D. J. Wexler, R. W. Grant, J. B. Meigs, D. M. Nathan, and E. Cagliero, "Sex disparities in treatment of cardiac risk factors in patients with type 2 diabetes," Diabetes Care, vol. 28, no. 3 , pp. 514-520, 2005.

[18] S. E. Back, R. L. Payne, A. H. Wahlquist et al., "Comparative profiles of men and women with opioid dependence: results from a national multisite effectiveness trial," The American Journal of Drug and Alcohol Abuse, vol. 37, no. 5, pp. 313-323, 2011.

[19] A. Setoodeh, F. Mostafavi, A. Rabbani, and T. Hedayat, "Female sex as a risk factor for glycemic control and 
complications in Iranian patients with type one diabetes mellitus," Iranian Journal of Pediatrics, vol. 21, no. 3, pp. 373-378, 2011.

[20] M. M. Levy, M. P. Fink, J. C. Marshall et al., "2001 SCCM/ ESICM/ACCP/ATS/SIS international sepsis definitions conference," Critical Care Medicine, vol. 31, no. 4, pp. 1250-1256, 2003.

[21] E. M. Bucholz, N. M. Butala, S. S. Rathore, R. P. Dreyer, A. J. Lansky, and H. M. Krumholz, "Sex differences in longterm mortality after myocardial infarction," Circulation, vol. 130, no. 9, pp. 757-767, 2014.

[22] J. G. Canto, M. G. Shlipak, W. J. Rogers et al., "Prevalence, clinical characteristics, and mortality among patients with myocardial infarction presenting without chest pain," JAMA, vol. 283, no. 24, pp. 3223-3229, 2000.

[23] D. Grady, L. Chaput, and M. Kristof, "Diagnosis and treatment of coronary heart disease in women: systematic reviews of evidence on selected topics," Evidence Report/Technology Assessment (Summary), vol. 81, pp. 1-4, 2003.

[24] S. E. Back, R. L. Payne, A. N. Simpson, and K. T. Brady, "Gender and prescription opioids: findings from the national survey on drug use and health," Addictive Behaviors, vol. 35, no. 11, pp. 1001-1007, 2010. 